\title{
The Metallicity dependence of the Cepheid Period-Luminosity relation: methodology and results
}

\author{
Marta Mottini ${ }^{1}$, Martino Romaniello ${ }^{1}$, Francesca Primas ${ }^{1}$, Martin \\ Groenewegen $^{2}$, Giuseppe Bono ${ }^{3}$, and Patrick François ${ }^{4}$ \\ 1 European Southern Observatory, Karl-Schwarzschild Strasse 2, D-85748 Garching \\ b. München, Germany \\ 2 Istituut voor Sterrenkunde, Celestijnenlaan 200B, B-3001 Leuven, Belgium \\ 3 INAF-Osservatorio Astronomico di Roma, via Frascati 33, I-00040 Monte Porzio \\ Catone, Italy \\ 4 Observatoire de Paris-Meudon, GEPI, 61 avenue de l'Observatoire, F-75014 Paris, \\ France
}

\begin{abstract}
We present the results of an observational campaign undertaken to assess the influence of the iron content on the Cepheid Period-Luminosity relation. Our data indicate that this dependence is not well represented by a simple linear relation. Rather, the behaviour is markedly non monotonic, with the correction peaking at about solar metallicity and declining for higher and lower values of $[\mathrm{Fe} / \mathrm{H}]$.
\end{abstract}

\section{Data analysis and results}

Cepheid stars, through their Period-Luminosity relation, are one of the pillars on which the extragalactic distance scale is built. To this day, however, the debate is still open on the role played by the chemical composition on the pulsational properties of these stars, with different theoretical models and observational results leading to markedly different conclusion (e.g. [1], [2], [4], [5]). To tackle this problem we used high resolution spectra collected with UVES and FEROS .Our sample includes a total of 76 stars: 40 Galactic, 22 LMC, and 14 SMC Cepheids. As first step in our analysis of the chemical composition of Cepheids we focused on the iron content. We developed a robust analysis procedure in order to accurately determine the iron abundance. First, we carefully assembled a reliable list of 263 iron lines (both neutral and ionized) between 480 and $780 \mathrm{~nm}$. We selected the oscillator strenght from the VALD database and we visually inspected each line profile on the observed spectra in order to detect and eliminate those lines affected by other elemental blends. Secondly, we measured the equivalent widths of all the lines assembled as described above using the FITLINE routine based on Gaussian fit. Finally, our iron abundances were determined using Kurucz's ATLAS9 model atmospheres and the WIDTH9 code ([6]).

We have determined the iron abundances for a sub-sample of stars: 13 Galactic, 13 LMC and 12 SMC Cepheids. Our main result is summarised in the Fig.1, where we plot the V-band residuals $\delta\left(M_{V}\right)$ of our stars from the standard PL relation of [3] as a function of the iron abundance we have derived from the 


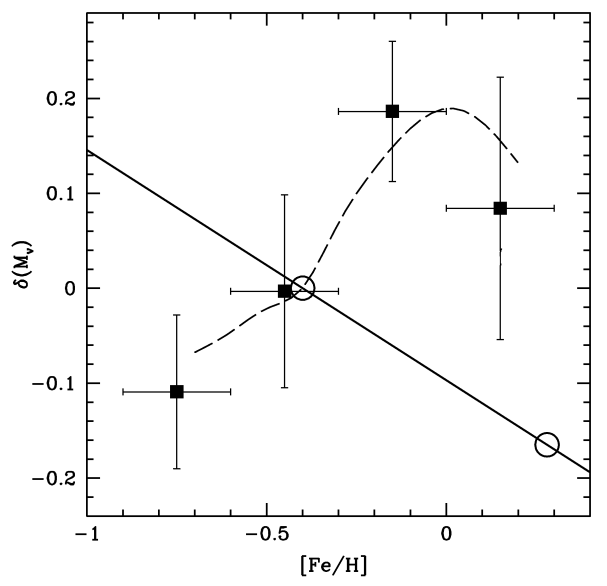

Fig. 1. Result from the subset analysed so far. The data are binned in metallicity to reflect the typical uncertainty on our determination of $[\mathrm{Fe} / \mathrm{H}]$, marked by horizontal error-bars. The median value of $\delta\left(M_{V}\right)$ in each metallicity bin is plotted as filled squares, with the vertical error-bars representing its associated error. For comparison we also plot the empirical results from [5] in two Cepheid fields in M101 (open circles and solid line) and the theoretical predictions by [2] from non-linear pulsational models (dashed line)

FEROS and UVES spectra. This standard PL relation was derived for the LMC as a whole $(<[\mathrm{Fe} / \mathrm{H}]>\cong-0.4)$ and $\delta\left(M_{V}\right)$ is, effectively, the correction to be applied to a "universal" PL relation as a function of metallicity. A positive $\delta\left(M_{V}\right)$ means fainter than the standard PL relation.

Our data indicate that the stars become fainter as metallicity increases, until a plateau or turnover point is reached at about solar metallicity. Our data are incompatible with both no dependence of th PL relation on iron abundance and with the linearly decreasing behaviour often found in the literature (e.g. [5], [8]). On the other hand, non-linear theoretical models of [2] provide a fairly good description of the data. For an in-depth discussion see [7].

\section{References}

1. I. Baraffe, Y. Alibert: A\&A 371, 592 (2001)

2. G. Fiorentino, F. Caputo, M. Marconi, I. Musella: ApJ 576, 402 (2002)

3. W.L. Freedman, B.F. Madore, B.K. Gibson et al: ApJ 553, 47 (2001)

4. A.M. Fry, B.W. Carney: AJ 113, 1073 (1997)

5. R.C. Kennicutt, P.B. Stetson, A. Saha, D. Kelson et al: ApJ 498, 181 (1998)

6. R.L. Kurucz: CD-ROMs \#1, 13, 18 (1993)

7. M. Romaniello, F. Primas, M. Mottini, M. Groenewegen, G.Bono, P. François: A\&A submitted (2004)

8. S. Sakai, L. Ferrarese, R.C. Kennicutt, A. Saha: ApJ 608, 42 (2004) 